\title{
Interface for Barge-in Free Spoken Dialogue System Based on Sound Field Reproduction and Microphone Array
}

\author{
Shigeki Miyabe, ${ }^{1}$ Yoichi Hinamoto, $^{2}$ Hiroshi Saruwatari,, ${ }^{1}$ Kiyohiro Shikano, ${ }^{1}$ and Yosuke Tatekura ${ }^{3}$ \\ ${ }^{1}$ Graduate School of Information Science, Nara Institute of Science and Technology, Takayama-Cho 8916-5, \\ Ikoma-Shi, Nara 630-0192, Japan \\ ${ }^{2}$ Department of Control Engineering, Takuma National College of Technology, Takuma-Cho Koda 551, Mitoyo-Shi, \\ Kagawa 769-1192, Japan \\ ${ }^{3}$ Faculty of Engineering, Shizuoka University, Johoku 3-5-1, Hamamatsu-Shi, Shizuoka 432-8561, Japan
}

Received 1 May 2006; Revised 17 October 2006; Accepted 29 October 2006

Recommended by Aki Harma

\begin{abstract}
A barge-in free spoken dialogue interface using sound field control and microphone array is proposed. In the conventional spoken dialogue system using an acoustic echo canceller, it is indispensable to estimate a room transfer function, especially when the transfer function is changed by various interferences. However, the estimation is difficult when the user and the system speak simultaneously. To resolve the problem, we propose a sound field control technique to prevent the response sound from being observed. Combined with a microphone array, the proposed method can achieve high elimination performance with no adaptive process. The efficacy of the proposed interface is ascertained in the experiments on the basis of sound elimination and speech recognition.
\end{abstract}

Copyright (c) 2007 Shigeki Miyabe et al. This is an open access article distributed under the Creative Commons Attribution License, which permits unrestricted use, distribution, and reproduction in any medium, provided the original work is properly cited.

\section{INTRODUCTION}

For hands-free realization of smooth communication with a spoken dialogue system, it should be guaranteed that a user's command utterance reaches the system clearly. However, a user might interrupt sound responses from the system and utter a command, or he might start speaking before the termination of the sound responses from the system. In such a situation, the sound given from the system to the user is observed as an acoustic echo return at a microphone used for acquisition of the user's speech input, and degrades the speech recognition performance in receiving the user's input command. Such a situation is referred to as barge-in [1]. Hereafter, the sound message outputted from the system is called response sound.

As a solution to this problem, an acoustic echo canceller is commonly used [2]. Since the echo return of the response sound is a convolution of the known response sound signal and a transfer function from a loudspeaker to a microphone, we eliminate the echo return by estimating the transfer function with an adaptive filter. Many types of acoustic echo canceller have been proposed, such as single-channel, stereophonic, beamformer-integrated, and wave-synthesis-integrated types [3-6]. The room transfer function is variable and fluctuates because of changes of room conditions, such as the movement of people in the room and changes in temperature [7]. Therefore, the adaptation must be continued even after its temporary convergence. However, in the state of barge-in (this is also called a "double-talk problem"), since user's speech input is mixed in the observed signal, the speech acts as noise to the estimation and the estimation fails. In this case, the adaptation process should be stopped by some type of double-talk detection technique $[8,9]$. Therefore, when the room transfer function changes in the barge-in state, the elimination performance degrades.

In order to achieve robustness, we propose a new interface for a barge-in free spoken dialogue system that combines multichannel sound field control and a microphone array. At first, to prevent the response sound from being observed at the microphone elements, we utilize the sound field reproduction technique via multiple loudspeakers and an inverse filter of the room transfer functions [10]. The sound field reproduction is generally used in a transaural system [11], which presents a three-dimensional sound image to a user at a fixed position. We apply this technique to the response 
sound elimination by controling sound field around the microphone to be silent alongside the transaural reproduction at user's ears. In the next step, user's speech is enhanced by microphone array signal processing. By increasing the numbers of loudspeakers and microphone elements, the control of the proposed method becomes robust against the fluctuation of the room transfer functions. With sufficient numbers of loudspeakers and microphones, the proposed method enables us to eliminate the response sound with enough robustness to sustain speech recognition accuracy.

Although the proposed method requires many loudspeakers and the cost for the hardware is higher than the conventional acoustic echo canceller, the proposed method uses a fixed filter designed in advance and real-time adaptation is unnecessary. As a result, computational cost can be reduced. In addition, the proposed method has an advantage that sound virtual reality [12] can be achieved with transaural reproduction. Thus we can realize duplex telecommunication, for example, video conference, with telepresence as if the users share the same space. Besides, we can apply the proposed method for control of car navigation system by spoken dialogue system. We can eliminate not only the response sound of the car navigation but also music of car audio. Moreover, in this case user's position is limited and nowadays car interior has many loudspeakers whose positions are fixed. Therefore the disadvantage of the proposed method, that is, fix of the positions of the loudspeakers and the user, is not problematic.

In Section 2, we describe the basic concept and problems of the conventional acoustic echo canceller. In Section 3, we describe the principle of the proposed interface. In Section 4, an experimental comparison of response sound elimination performances is carried out. In Section 5, the effectiveness of the proposed method is validated in the speech recognition experiment. In Section 6, we assess the quality of the response sound reproduced by the proposed method.

\section{CONVENTIONAL ACOUSTIC ECHO CANCELLER}

To eliminate the acoustic echo of the response sound, an acoustic echo canceller is generally used. In this section, we describe the basic principle of the acoustic echo canceller, and indicate its weakness against the fluctuation of a room transfer function.

\subsection{Principle and problem of conventional acoustic echo canceller}

The configuration of an acoustic echo canceller using an adaptive filter is shown in Figure 1. Let the source signal of the response sound be $x(\omega)$, where $\omega$ shows the angular frequency. The echo return of the response sound $y_{\text {mic }}(\omega)$ can be written as the product of $x(\omega)$ and the transfer function $g_{\text {mic }}(\omega)$ from a loudspeaker to a microphone,

$$
y_{\text {mic }}(\omega)=g_{\text {mic }}(\omega) x(\omega) .
$$

The acoustic echo canceller calculates an estimate $g_{\text {mic }}(\omega)$, denoted as $\hat{g}_{\text {mic }}(\omega)$. Then the estimated response sound

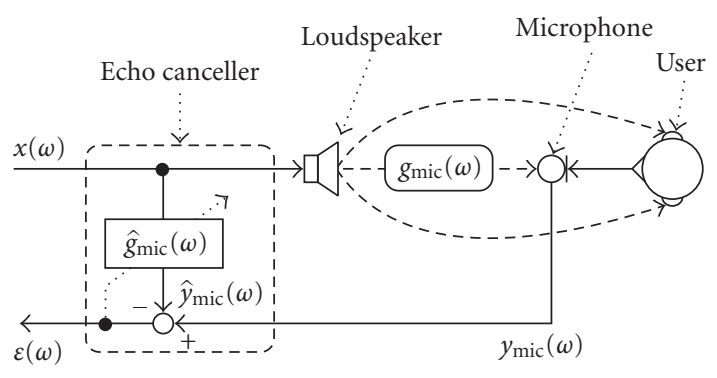

FIgURE 1: Configuration of acoustic echo canceller in spoken dialogue system.

$\hat{y}_{\text {mic }}(\omega)$ can be obtained as

$$
\hat{y}_{\text {mic }}(\omega)=\hat{g}_{\text {mic }}(\omega) x(\omega) .
$$

To estimate $g_{\text {mic }}(\omega)$, an adaptive filter is used and the estimated transfer function $\hat{g}_{\text {mic }}(\omega)$ is updated iteratively to minimize the power of the error signal $\epsilon(\omega)$,

$$
\epsilon(\omega)=y_{\text {mic }}(\omega)-\hat{y}_{\text {mic }}(\omega) .
$$

Once the room transfer function is estimated, the acoustic echo canceller can eliminate the response sound sufficiently. However, whenever the transfer function is changed, it must be reestimated. To follow the fluctuation of the transfer function in real time, online adaptation, for example, least mean squares [13] or recursive least squares, is used. However, these adaptation techniques are weak against noise. In the state of barge-in, since user's input speech is mixed with the observed signal, an accurate error of the estimation cannot be obtained and the adaptation diverges. Therefore, the adaptation must be stopped using double-talk detection [8]. However, it is often difficult to decide whether the error is caused by either fluctuation or barge-in.

\subsection{Response sound elimination error of the acoustic echo canceller when fluctuation of the room transfer function occurs}

The room transfer functions are easily changed with the variation of the system's state such as the movement of people. In this section, the response sound elimination error signal $\epsilon(\omega)$ is examined in the case where the transfer function is changed. Suppose that the variation $\Delta g_{\text {mic }}(\omega)$ caused by the fluctuation of room transfer functions is added to the original transfer function $g_{\text {mic }}(\omega)$. In this case, the response sound is expressed as

$$
y_{\text {mic }}(\omega)=\left[g_{\text {mic }}(\omega)+\Delta g_{\text {mic }}(\omega)\right] x(\omega) .
$$

The elimination error signal $\epsilon(\omega)$ of the response sound is written using the estimated filter $\widehat{g}_{\text {mic }}(\omega)$ as

$$
\epsilon(\omega)=\Delta g_{\mathrm{mic}}(\omega) x(\omega),
$$

where we assume that the filter was exactly estimated so as to satisfy $\hat{g}_{\text {mic }}(\omega)=g_{\text {mic }}(\omega)$ and $g_{\text {mic }}(\omega) x(\omega)-\hat{g}_{\text {mic }}(\omega) x(\omega)=0$. 


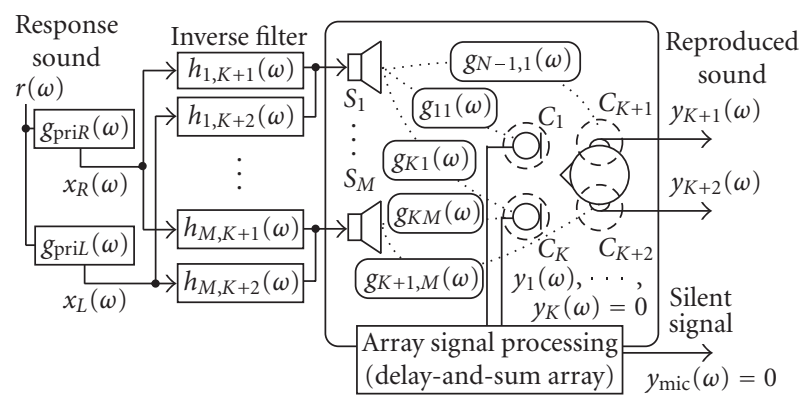

Figure 2: Configuration of the proposed system.

Since the acoustic echo canceller has no mechanism for improving the robustness of the elimination (unless it contains a suitable post-processing for that case), the fluctuation of the transfer function effects directly its error. Therefore, if the fluctuation occurs when the adaptation stops because of barge-in, its elimination performance degrades.

\section{PROPOSED METHOD: MULTIPLE-OUTPUT AND MULTIPLE-NO-INPUT METHOD}

In this section, we propose a new response sound elimination technique, which is robust against the fluctuation of the room transfer function. The proposed method mainly consists of two steps. First, sound field control with multiple loudspeakers realizes silent zones at the microphone elements while the dialogue system gives the response sound to the user. Next, by delay-and-sum-type signal processing using a microphone array, the residual component of the response sound caused by the fluctuation of the transfer function is suppressed and user's utterance is emphasized. The response sound signal is outputted from the multiple loudspeakers and cancelled at multiple control points. With this mechanism, the response sound is prevented from being inputted to the speech recognition system. Thus we call this technique multiple-output/multiple-no-input (MOMNI) method. We discuss the relation between the robustness of the control and the number of transfer channels. Then it is proved that we can improve its robustness against the fluctuation of the transfer functions by increasing the numbers of loudspeakers and microphone elements. With sufficient numbers of loudspeakers and microphones, the MOMNI method can eliminate the response sound with enough robustness using fixed filter coefficients. Needless to say, this processing requires no double-talk detection.

\subsection{Sound field control}

Here, we describe the sound field control used to eliminate the acoustic echo of the response sound from the system. The configuration of the proposed system is shown in Figure 2. Let $M$ be the number of secondary sound sources $S_{1}, \ldots, S_{M}$ and let $N$ be the number of control points $C_{1}, \ldots, C_{N}$. The control points $C_{1}, \ldots, C_{K}(K=N-2)$ are arranged to the elements of a microphone array for acquisition of user's speech, and $C_{K+1}$ and $C_{K+2}$ are set at both ears of the user. The signals to be reproduced at the control points $C_{1}, \ldots, C_{K+2}$ are described by

$$
\mathbf{x}(\omega)=\left[x_{\operatorname{mic} 1}(\omega), \ldots, x_{\operatorname{mic} K}(\omega), x_{\mathrm{R}}(\omega), x_{\mathrm{L}}(\omega)\right]^{\mathrm{T}},
$$

and similarly, the signals observed at these control points are represented by

$$
\mathbf{y}(\omega)=\left[y_{\text {mic } 1}(\omega), \ldots, y_{\operatorname{mic} K}(\omega), y_{\mathrm{R}}(\omega), y_{\mathrm{L}}(\omega)\right]^{\mathrm{T}}
$$

Using, for example, chirp signal [14], we should measure in advance all of the transfer functions from secondary sound sources $S_{m}$ to control points $C_{n}$, denoted by $g_{n m}(\omega)$, where $n=1, \ldots, N$, and $m=1, \ldots, M$. Here, to design an inverse filter of the transfer functions with nonminimum phases, the condition $M>N$ must hold [10]. To use fixed filter coefficients for the inverse filter, the positions of the loudspeakers and the microphones should not be changed after the measurement. In addition, we specify the position for the user to listen to the response sound, by, for example, setting a chair at the position. Here in the phase of the measurement, to obtain the transfer function of user's ears, since it is a burden for the user to sit on the position wearing microphones at his/her ears, we can substitute the user by a head and torso simulator (HATS) with microphones at the ears. Let $\mathbf{G}(\omega)$ be an $N \times M$ matrix consisting of $g_{n m}(\omega)$, and let $\mathbf{H}(\omega)$ be an $M \times N$ inverse filter matrix with components $h_{m k}(\omega)$. The inverse filter $\mathbf{H}(\omega)$ is then designed so that

$$
\mathbf{G}(\omega) \mathbf{H}(\omega)=\mathbf{I}_{N}(\omega),
$$

where $\mathbf{I}_{N}(\omega)$ denotes an $N \times N$ identity matrix. Using the transfer function matrix $\mathbf{G}(\omega)$ and the inverse filter matrix $\mathbf{H}(\omega)$, the relation between the observed signals $\mathbf{y}(\omega)$ and the reproduced signals $\mathbf{x}(\omega)$ is written as

$$
\mathbf{y}(\omega)=\mathbf{G}(\omega) \mathbf{H}(\omega) \mathbf{x}(\omega)
$$

In (9), we reproduce the response sounds of a dialogue system at both the user's ears (i.e., $\left[y_{R}(\omega), y_{L}(\omega)\right]=\left[x_{R}(\omega)\right.$, $\left.\left.x_{\mathrm{L}}(\omega)\right]\right)$, and reproduce silent signals with zero amplitudes at the microphone elements (i.e., $\left[y_{\operatorname{mic} 1}(\omega), \ldots, y_{\operatorname{mic} K}(\omega)\right]=$ $[0, \ldots, 0])$ as

$$
\mathbf{x}(\omega)=[\underbrace{0, \ldots, 0}_{K}, x_{\mathrm{R}}(\omega), x_{\mathrm{L}}(\omega)]^{\mathrm{T}} .
$$

By this sound reproduction, we can actualize a sound field in which the response sound is presented to the user while the response sound cancels at the microphone elements.

To remove the redundant filtering process of the zero signals, we truncate the matrix $\mathbf{H}(\omega)$ into $\mathbf{H}^{\prime}(\omega)$ which is an $M \times 2$ filter matrix composed of the filter components $h_{m k^{\prime}}(\omega)\left(m=1, \ldots, M, k^{\prime}=K+1, K+2\right)$ which are taken from $\mathbf{H}(\omega)$. By inputting the response sound to this filter matrix, the following equation holds:

$$
\begin{aligned}
\mathbf{y}(\omega) & =\mathbf{G}(\omega) \mathbf{H}^{\prime}(\omega)\left[x_{\mathrm{R}}(\omega), x_{\mathrm{L}}(\omega)\right]^{\mathrm{T}} \\
& =[\underbrace{0, \ldots, 0}_{K}, x_{\mathrm{R}}(\omega), x_{\mathrm{L}}(\omega)]^{\mathrm{T}} .
\end{aligned}
$$


Therefore, the condition equivalent to (10) can be realized with an $M \times 2$ filter matrix.

Since the proposed method uses an inverse filter of the room transfer function, we can show the response sound to the user in the form of a transaural system, say, a threedimensional sound field localization [11]. In transaural system, we can show the user a clear sound image of a primary sound source by reproducing a binaural signal [15], say, a convolution of a signal and transfer functions from the sound source to a person's ears. To provide a practical application of this property, we generate the response sound signals $x_{\mathrm{R}}(\omega)$ and $x_{\mathrm{L}}(\omega)$ by multiplying a monaural source of the response sound signal $r_{\mathrm{src}}(\omega)$ and the room transfer functions $\mathbf{g}_{\text {pri }}(\omega)=\left[g_{\text {priR }}(\omega), g_{\text {priL }}(\omega)\right]^{\mathrm{T}}$ between a primary sound source and both the user's ears as

$$
\left[x_{\mathrm{R}}(\omega), x_{\mathrm{L}}(\omega)\right]^{\mathrm{T}}=\mathbf{g}_{\text {pri }}(\omega) r_{\text {src }}(\omega) .
$$

In the transaural reproduction described above, the sound image is degraded when the user is not at the prepared position because the perceived response sound is not an accurate binaural sound. However, the sound quality away from the prepared position is sufficient for the presentation of the response sound for the spoken dialogue system. We will justify this argument in the experiment in Section 6.

\subsection{Signal processing using microphone array}

In this section, we will focus our attention on array signal processing. In this study, we adopt a delay-and-sum array signal processing [16] to emphasize the user's utterance. The filter of the $k$ th element in the delay-and-sum array is denoted by $w_{k}(\omega)$ for $k=1, \ldots, K$. Then $w_{k}(\omega)$ can be expressed as

$$
w_{k}(\omega)=\frac{1}{K} \cdot e^{-j \omega \tau_{k}},
$$

where $\tau_{k}$ stands for the arrival time difference of the user's utterance between a suitable standard point and the $k$ th element position. We set $\tau_{k}$ to form a directivity to the look direction of the user. Suppose that the signal added through the array filters is a signal for speech recognition. Then the response sound contained in the observed signal is expressed as

$$
y_{\text {mic }}(\omega)=\sum_{k=1}^{K} w_{k}(\omega) y_{\text {mic } k}(\omega) .
$$

When this delay-and-sum-type array is used, the system's response sounds which arrive from other than the target direction are out of phase at each element, and only the user's speech which comes from the target direction is in phase at each element and is added. As a result, only user's speech can be emphasized in the $y_{\text {mic }}(\omega)$. Thus we give this signal to the speech decoder to recognize the user's speech.

\subsection{Inverse system design for sound field reproduction}

In a multipoint control system which controls multiple control points with many loudspeakers, large amounts of calculation and memory are needed to design an inverse filter in the time domain. Therefore, we design the inverse filter matrix $\mathbf{H}(\omega)$ by using the least-norm solution (LNS) in the frequency domain [12]. The method has advantages that the amount of calculation is small in the frequency domain, and the designed system is stable because the output from each sound source is suppressed to the minimum. Here, we use the Moore-Penrose generalized inverse matrix as the inverse matrix which gives the least-norm solution. We obtain a singular value decomposition of $\mathbf{G}(\omega)$ as

$$
\begin{gathered}
\mathbf{G}(\omega)=\mathbf{U}(\omega)\left[\boldsymbol{\Gamma}_{N}(\omega), \mathbf{O}_{N, M-N}(\omega)\right] \mathbf{V}^{\mathrm{H}}(\omega), \\
\boldsymbol{\Gamma}_{N}(\omega) \equiv \operatorname{diag}\left[\mu_{1}(\omega), \mu_{2}(\omega), \ldots, \mu_{N}(\omega)\right],
\end{gathered}
$$

where $\mathbf{U}(\omega)$ and $\mathbf{V}(\omega)$ are $N \times N$ and $M \times M$ unitary matrices, respectively, $\mu_{n}(\omega)$ for $n=1,2, \ldots, N$ are the singular values of $\mathbf{G}(\omega)$, and are arranged so that $\mu_{n}(\omega) \geq \mu_{n+1}(\omega)$ in matrix $\boldsymbol{\Gamma}_{N}(\omega), \mathbf{O}_{N, M-N}(\omega)$ denotes an $N \times(M-N)$ null matrix, and $\{\cdot\}^{\mathrm{H}}(\omega)$ represents a conjugate transposition.

Then the Moore-Penrose generalized inverse matrix $\mathbf{G}^{+}(\omega)(=\mathbf{H}(\omega))$ of $\mathbf{G}(\omega)$ is given by

$$
\begin{gathered}
\mathbf{G}^{+}(\omega)=\mathbf{V}(\omega)\left[\begin{array}{c}
\boldsymbol{\Lambda}_{N}(\omega) \\
\mathbf{O}_{M-N, N}(\omega)
\end{array}\right] \mathbf{U}^{\mathrm{H}}(\omega), \\
\boldsymbol{\Lambda}_{N}(\omega) \equiv \operatorname{diag}\left[\frac{1}{\mu_{1}(\omega)}, \frac{1}{\mu_{2}(\omega)}, \ldots, \frac{1}{\mu_{N}(\omega)}\right] .
\end{gathered}
$$

Then we utilize the Moore-Penrose generalized inverse matrix for the inverse filter as $\mathbf{H}(\omega)=\mathbf{G}^{+}(\omega)$.

\subsection{Response sound elimination error for fluctuation of room transfer functions}

In an acoustic echo canceller, because we need to reestimate the transfer function when it is changed, there is a problem that the response sound elimination accuracy degrades during the estimation process. In contrast, it is proved that the proposed technique is robust against the fluctuation of room transfer functions, even when the fixed filter coefficients are used. Here, we suppose that an inverse filter matrix computed before the fluctuation is used to control the sound field.

Supposing that the variation $\Delta g_{n m}(\omega)$ caused by the fluctuation of transfer functions is added to a transfer function $g_{n m}(\omega)$, the transfer function matrix after the fluctuation will become $\mathbf{G}(\omega)+\Delta \mathbf{G}(\omega)$, where $\Delta \mathbf{G}(\omega)$ is an $N \times M$ matrix composed of $\Delta g_{n m}(\omega)$. Then, by using an inverse filter matrix $\mathbf{H}(\omega)$ designed before the fluctuation of transfer functions, the signals $\mathbf{y}(\omega)$ observed at each control point are expressed as

$$
\begin{aligned}
\mathbf{y}(\omega) & =[\mathbf{G}(\omega)+\Delta \mathbf{G}(\omega)] \mathbf{H}(\omega) \mathbf{x}(\omega) \\
& =\left[\mathbf{I}_{N}(\omega)+\Delta \mathbf{G}(\omega) \mathbf{H}(\omega)\right] \mathbf{x}(\omega),
\end{aligned}
$$

and the errors caused by the fluctuation are represented as $\Delta \mathbf{G}(\omega) \mathbf{H}(\omega) \mathbf{x}(\omega)$. In this case, the error $\Delta y_{\text {mic }}(\omega)$ of the 
response sound elimination $y_{\mathrm{mic}}(\omega)$ in $(14)$ is written as

$$
\begin{aligned}
\Delta y_{\text {mic }} & (\omega) \\
= & \sum_{k=1}^{K} w_{k}(\omega) \\
& \times\left\{\sum_{m=1}^{M} \Delta g_{(k+2) m}(\omega) \cdot\left[h_{m 1}(\omega) x_{\mathrm{R}}(\omega)+h_{m 2}(\omega) x_{\mathrm{L}}(\omega)\right]\right\} .
\end{aligned}
$$

Since this system controls $y_{\text {mic }}(\omega)$ such that it is 0 before the fluctuation of transfer functions, $\Delta y_{\text {mic }}(\omega)$ after the fluctuation is the response sound elimination error signal $\epsilon(\omega)$. This is expressed as

$$
\epsilon(\omega)=y_{\mathrm{mic}}(\omega)+\Delta y_{\mathrm{mic}}(\omega)=\Delta y_{\mathrm{mic}}(\omega)
$$

Next, let the singular values of $\mathbf{G}(\omega)$ be $\mu_{j}(\omega)$ for $j=$ $1,2, \ldots, N$ and let the eigenvalues of $\mathbf{G}^{\mathrm{H}}(\omega) \mathbf{G}(\omega)$ be $\lambda_{j}(\omega)$ for $j=1,2, \ldots, N$. Then, the norm $\|\mathbf{G}(\omega)\|$ is given by

$$
\begin{aligned}
\|\mathbf{G}(\omega)\| & =\sqrt{\max _{j}\left(\lambda_{j}(\omega)\right)}=\sqrt{\max _{j}\left(\left\{\mu_{j}(\omega)\right\}^{2}\right)} \\
& =\left|\mu_{1}(\omega)\right|
\end{aligned}
$$

where $\max _{j}\left(a_{j}\right)$ denotes the largest element of $a_{j}$ for any $j$. The relation $\lambda_{j}(\omega)=\left\{\mu_{j}(\omega)\right\}^{2}$ is used here.

Alternatively, since the singular values of $\mathrm{G}^{+}(\omega)$ are given by $1 / \mu_{j}(\omega)$, the norm $\left\|\mathbf{G}^{+}(\omega)\right\|$ is expressed as

$$
\begin{aligned}
\left\|\mathbf{G}^{+}(\omega)\right\| & =\sqrt{\max _{j}\left(\frac{1}{\lambda_{j}(\omega)}\right)}=\sqrt{\max _{j}\left(\frac{1}{\left\{\mu_{j}(\omega)\right\}^{2}}\right)} \\
& =\frac{1}{\left|\mu_{N}(\omega)\right|} .
\end{aligned}
$$

Since the secondary sound source is arranged with almost equal distance for each control point, if the number of secondary sound sources, $M$, increases, the norm of $\mathbf{G}(\omega)$ is directly proportional to $M$, that is, $\|\mathbf{G}(\omega)\| \propto M$. Moreover, the condition number of $\mathbf{G}(\omega)$, which is expressed by the ratio between the maximum and minimum singular values, that is,

$$
\operatorname{cond}(\mathbf{G})=\frac{\mu_{1}}{\mu_{N}}
$$

is known to be close to unity when the number of secondary sound sources arranged is much larger than that of control points (this is experimentally proven in Section 4.3). Therefore, the following relation can be derived from (20) and (21):

$$
\begin{aligned}
\|\mathbf{H}(\omega)\| & =\left\|\mathbf{G}^{+}(\omega)\right\|=\frac{1}{\left|\mu_{N}(\omega)\right|} \\
& \simeq \frac{1}{\left|\mu_{1}(\omega)\right|}=\frac{1}{\| \mathbf{G}(\omega) \mid} \propto \frac{1}{M} .
\end{aligned}
$$

Substituting (13) into (18), we obtain

$$
\begin{aligned}
& \Delta \hat{y}_{\text {mic }}(\omega) \\
& =\|\mathbf{H}(\omega)\| \frac{1}{K}\left\{\sum_{k=1}^{K} \sum_{m=1}^{M} \Delta g_{k m}(\omega)\right. \\
& \left.\cdot\left[\bar{h}_{m(K+1)}(\omega) x_{\mathrm{R}}(\omega)+\bar{h}_{m(K+2)}(\omega) x_{\mathrm{L}}(\omega)\right] \cdot e^{-j \omega \tau_{k}}\right\},
\end{aligned}
$$

where $\bar{h}_{m n}(\omega)=h_{m n}(\omega) /\|\mathbf{H}(\omega)\|$. We assume that $\Delta g_{n m}(\omega)$ for $n=1,2, \ldots, N$ and $m=1,2, \ldots, M$ are mutually independent and follow the same Gaussian distribution with zero mean and variance $\sigma^{2}$. Furthermore, since $\bar{h}_{m n}(\omega)$ is a function normalized by $\|\mathbf{H}(\omega)\|$ and independent on $M$, the deviation of $\{\cdot\}$ in (24) can be represented by $\eta \sqrt{M K} \sigma$, where $\eta$ is a suitable constant. Therefore, the elimination error $\epsilon(\omega)$ of response sound is obtained from (23) as

$$
\epsilon(\omega)=\Delta y_{\mathrm{mic}}(\omega) \propto \frac{1}{M} \cdot \frac{1}{K} \cdot \sqrt{M K}=\frac{1}{\sqrt{M K}} .
$$

In other words, (25) shows that the elimination error of the response sound for the fluctuation of the transfer functions is inversely proportional to $\sqrt{M K}$. Thus, if the number of transfer channels from loudspeakers to microphones increases, the response sound elimination of the proposed method improves its robustness against the fluctuation of the transfer functions.

We remark that in the real environment, it is difficult to prove whether or not the variations $\Delta g_{n m}(\omega)$ caused by the fluctuation of the room transfer functions are mutually independent for every channel from a loudspeaker to a microphone. However, in the next section, the simulations using impulse responses measured in the real environment show that the error estimation in (25) is valid.

\section{EXPERIMENTAL COMPARISON OF RESPONSE SOUND ELIMINATION PERFORMANCE}

To assess the robustness of the proposed method against the fluctuation of the room transfer functions, the response sound elimination performance of the proposed method is evaluated by simulations. Its performance is compared with that of conventional acoustic echo canceller.

\subsection{Experimental conditions}

The simulations are carried out by using impulse responses measured in a real acoustic environment. Figure 3 shows the arrangement of the apparatuses. To imitate the user at the center of the room, we set a HATS. To cause fluctuations of the room transfer functions intentionally, we placed a lifesize mannequin as an interference near a user, under the assumption that a person approaches to the user. We measured in a total of 13 patterns of the room impulse responses: 12 patterns are for the state in which the interference is allocated, and the remaining pattern is for the state in which no 
interference exists. The transfer functions before fluctuation are used to design filters for both the acoustic echo canceller and the proposed method, and we evaluated the performance under static transfer functions after fluctuations. To prevent the effect of the change of condition to observe the user's utterance, we did not change the user's position in these fluctuations. A loudspeaker set in front of the user is used both as an acoustic echo canceller and as a primary sound source of the proposed method. The reverberation time is about 160 milliseconds. The room impulse responses are sampled at a frequency of $48 \mathrm{kHz}$ and the magnitudes are quantized to 16 bits. We used a circular array with 12 elements, and equally spaced elements were selected for use.

\subsubsection{Conventional acoustic echo canceller}

Our interest is focused on the robustness against the fluctuation of room transfer functions. Therefore, the experiment is carried out under the assumption that the filter coefficients of the acoustic echo canceller are once estimated precisely, and then the fluctuation occurs when the estimation stops because of barge-in. To imitate this situation, we used the transfer function before fluctuation as the estimated transfer function of the acoustic echo canceller, and fixed its filter coefficients. The microphone element closest to the user is chosen as a microphone for acquisition of the user's speech.

\subsubsection{Proposed method}

The inverse filter in the proposed method is calculated using only the impulse responses in the case where there is no fluctuation. The design conditions of the inverse filters are as follows: the number of secondary sound sources $M=4$ to 36 , the number of control points $N=3$ to 8 , the filter length 16384 , and the passband range 150 to $4000 \mathrm{~Hz}$.

\subsection{Evaluation score}

The response sound elimination performance is evaluated using echo return loss enhancement (ERLE) as

$$
\operatorname{ERLE}(\mathrm{dB})=10 \log _{10} \frac{\sum_{\omega}\left\{y_{\text {micref }}(\omega)\right\}^{2}}{\sum_{\omega}\{\epsilon(\omega)\}^{2}},
$$

where $y_{\text {micref }}(\omega)$ is the response sound reproduced at a standard microphone, and $\epsilon(\omega)$ is the response sound elimination error signal derived from (5) or (19).

\subsection{Experimental results and discussion}

Figures 4-6 show that frequency characteristics of the response sound elimination error signal in the conventional acoustic echo canceller and proposed method after the room transfer function have changed. In these evaluations, we used a female utterance selected from the ASJ database [17] as a response sound. From these figures, it turns out that the response sound can be suppressed independent of frequency in the passband by even which techniques.

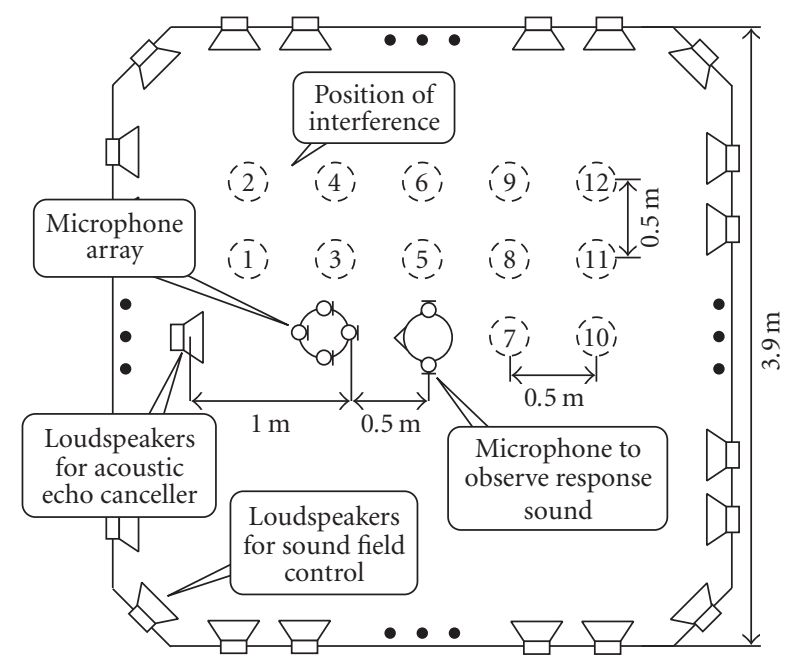

FIGURE 3: Layout of acoustic experiment room.

The ERLE for each position of the interference in the case of the typical number of loudspeakers and 2 elements is shown in Figure 7, and that for each position of interference in the case of 24 loudspeakers and the typical number of microphones is in Figure 8. In these evaluations, to remove the effect of the bias of frequency characteristics, we used a white noise as a response sound. It can be seen that increasing both the number of microphone elements and the number of loudspeakers improves the performance of the proposed method, and can make the control robust against the fluctuation of room transfer functions. Regardless of the position of the interference, the performance of the proposed method is superior to that of the conventional echo canceller. Hereafter, we discuss only the averaged ERLE of 12 types of fluctuations.

In Figure 9, ERLE is shown as a function of the number of transfer channels $(=M K)$ from the loudspeakers to the microphone elements. The theoretical curve in the figure is drawn by plotting the ERLE derived from (25), which is given by

$$
\begin{aligned}
\operatorname{ERLE}_{\text {theory }}(\mathrm{dB}) & =10 \log _{10} \frac{\sum_{\omega}\left\{y_{\text {micref }}(\omega)\right\}^{2}}{\sum_{\omega}\{\epsilon(\omega)\}^{2}} \\
& =10 \log _{10} \frac{\sum_{\omega}\left\{y_{\text {mic }}(\omega)\right\}^{2}}{\sum_{\omega}\left\{\Delta \hat{y}_{\text {mic }}(\omega)\right\}^{2}} \\
& \propto \xi+10 \log _{10} \frac{1}{1 /(M K)} \\
& \propto \xi+10 \log _{10}(M K),
\end{aligned}
$$

where $\xi$ is a suitable constant.

From this figure, we can see that the response sound elimination performance is improved if the number of transfer channels increases. It also turns out that the deviation between the experimental and theoretical values arises when the number of microphone elements increases. The reasons are as follows. 


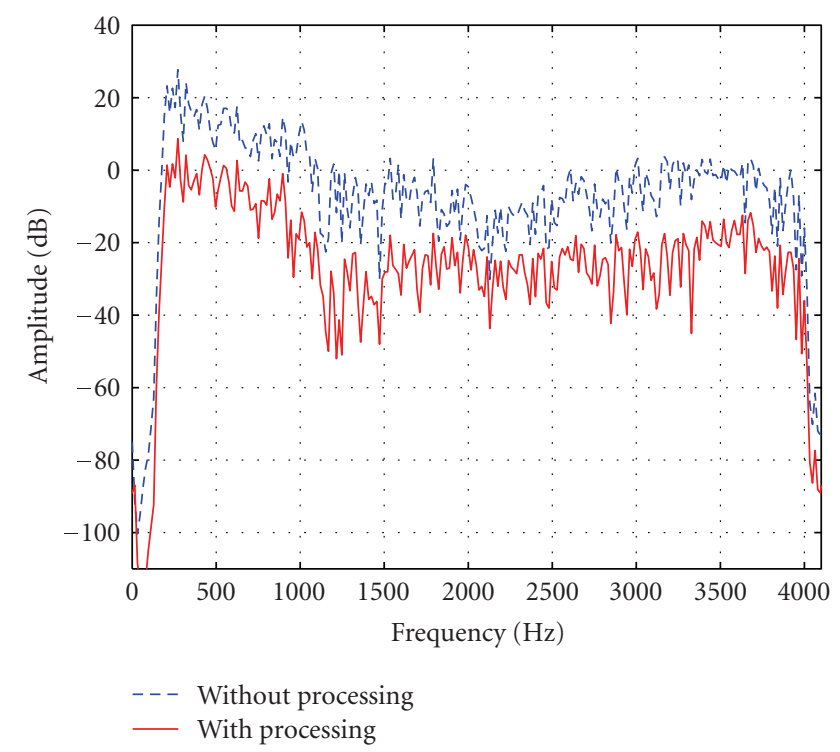

FIGURE 4: Example of frequency characteristics of observed signal obtained by acoustic echo canceller. The signal is observed at the microphone near the user. The position of interference is number 1 in Figure 3.

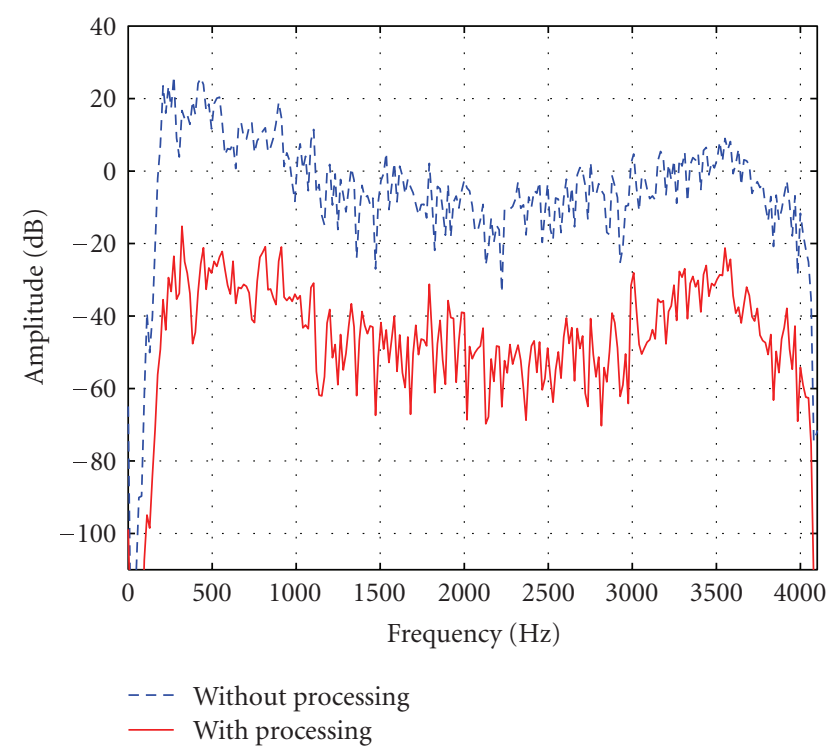

FIGURE 5: Example of frequency characteristics of observed signal obtained by the proposed method with 36 loudspeakers and $1 \mathrm{mi}$ crophone element. The signal is observed at the microphone near the user. The position of interference is number 1 in Figure 3.

(A) The stability margin of the inverse filters becomes small when the number of control points is close to that of the secondary sound sources.

(B) When there exist too many transfer channels, the independence of each channel is no longer valid. Consequently, the performance is saturated.

To prove the above claim (A), we show the condition number of transfer functions in Figure 10. The condition

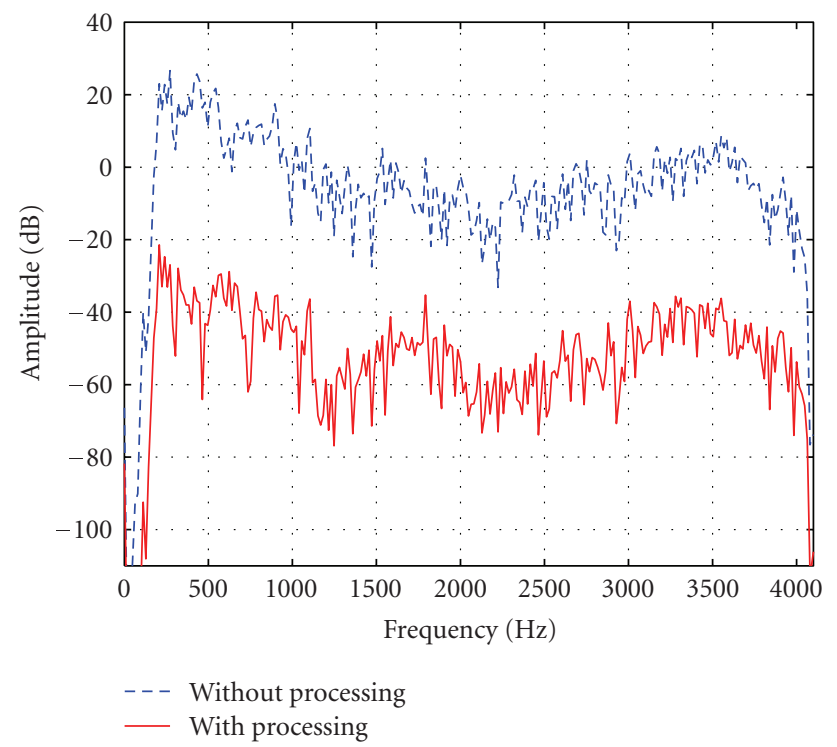

FIgURE 6: Example of frequency characteristics of observed signal obtained by proposed method with 36 loudspeakers and 6 microphone elements. The signal is observed at the microphone near the user. The position of interference is number 1 in Figure 3.

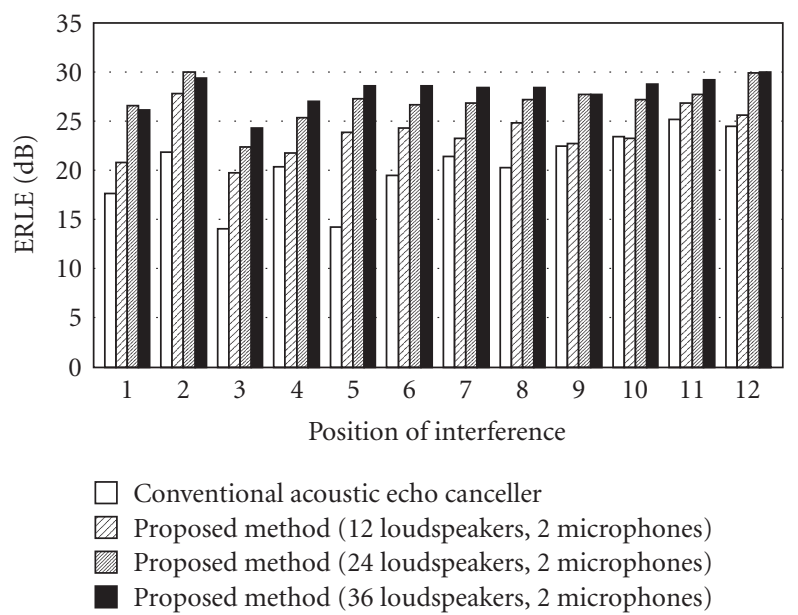

FIGURE 7: ERLE for each position of interference in 2 microphone elements. The horizontal axis represents the position of interference in Figure 3.

number, expressed as cond $(\mathbf{G}(\omega))$ in (22), represents the unstableness of the inverse filters. This figure shows that the condition number becomes close to 1 when the number of loudspeakers is much larger than that of the microphone elements (equal to the number of control points minus two), as argued in Section 3.4. However, when the number of microphone elements increases, the condition number increases. In addition, such a tendency becomes remarkable when the number of the secondary sound sources is small. This causes an appreciable degradation in ERLE.

Comparing the conventional acoustic echo canceller with the proposed method in Figure 9, we see that the proposed 


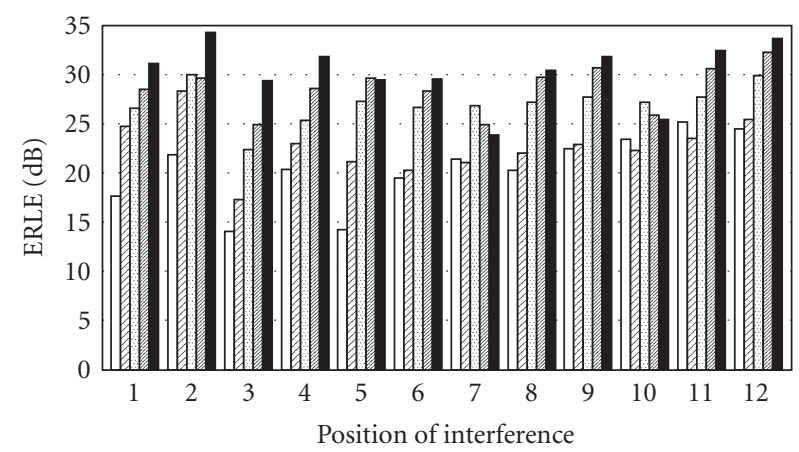

$\square$ Conventional acoustic echo canceller

Proposed method (24 loudspeakers, 1 microphone)

Proposed method (24 loudspeakers, 2 microphones)

20 Proposed method (24 loudspeakers, 4 microphones)

Proposed method (24 loudspeakers, 6 microphones)

FIGURE 8: ERLE for each position of interference in 24 loudspeakers. The horizontal axis represents the position of interference in Figure 3.

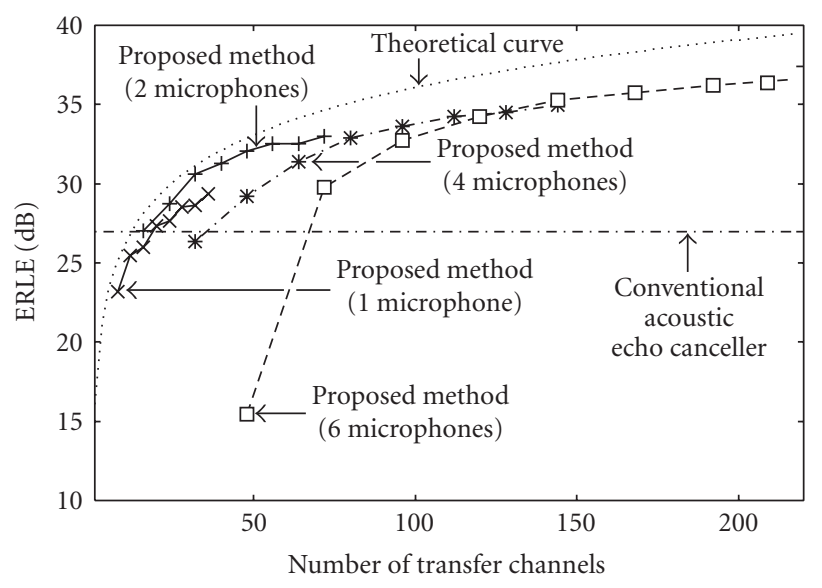

FIGURE 9: ERLE for different numbers of room transfer channels from loudspeakers to microphone elements.

method is more robust against the fluctuation of transfer functions if the number of transfer channels increases.

\section{SPEECH RECOGNITION EXPERIMENT}

The experiment involving large vocabulary speech recognition is carried out to investigate the efficacy of the proposed method, compared to that of the conventional acoustic echo canceller.

\subsection{Experimental conditions}

In the recognition experiment, we use the speech signal obtained by imposing the response sound elimination error signal $\epsilon(\omega)$ on the user's input speech. A large vocabulary recognition engine Julius ver. 3.4.2 [18] is used as a speech

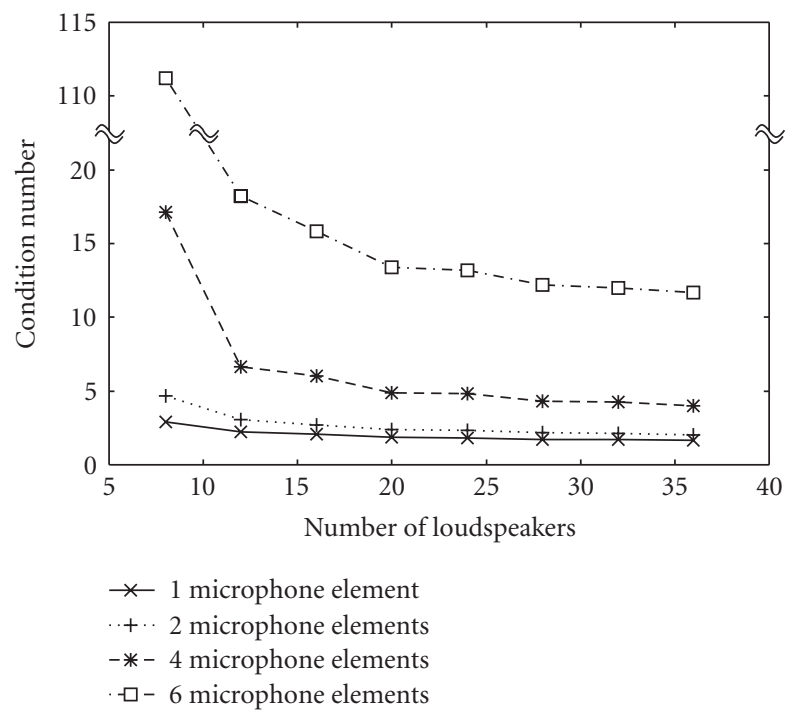

Figure 10: Condition number of average in passband.

decoder. We used two kinds of speaker-independent phonetic tied mixtures [19] as phoneme models. One is an ordinary clean model. The other is generated by a known-noise imposition technique [20] (see the appendix). We imposed a known noise of $30 \mathrm{~dB}$ on the observed signals to mask the redundant response sound, and to match its phoneme features, we imposed the noise of $25 \mathrm{~dB}$ on the speech in the learning data. A language model is made from newspaper dictation with a vocabulary of 20000 words [21]. As the user's speech, 200 sentences obtained from 23 males and 23 females are used through the JNAS database [22]. As the response sound of the dialogue system, a sentence of a female's speech from the ASJ database is used. Experimental conditions such as interference arrangements to cause changes of the transfer functions are the same as in the previous section.

\subsection{Evaluation score}

In order to evaluate the speech recognition performance, we adopt the word accuracy as an evaluation score. Word accuracy is defined as follows:

$$
\operatorname{word} \operatorname{accuracy}(\%)=\frac{W-S-D-I}{W},
$$

where $W$ is the total number of words in the test speech, $S$ is the number of substitution errors, $D$ is the number of deletion errors, and I is the number of insertion errors. The resultant recognition score is computed using the average value of data derived from the 200 sentences.

\subsection{Experimental results and discussions}

The speech recognition results obtained by the proposed method are shown in Figure 11 for the clean model, and in Figure 12 for the known-noise imposition. The results of the recognition experiment show that the word accuracy is 


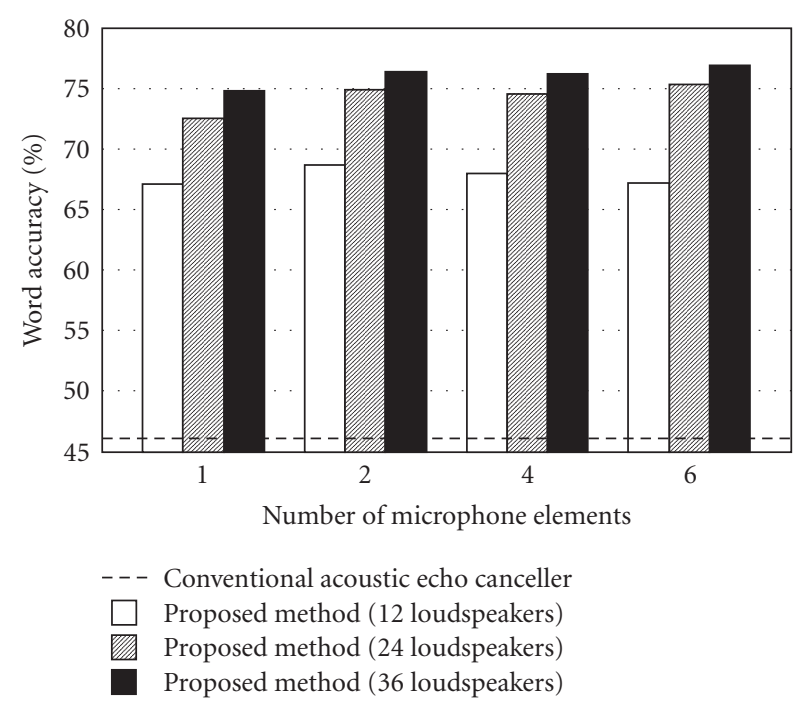

Figure 11: Word accuracy with clean model.

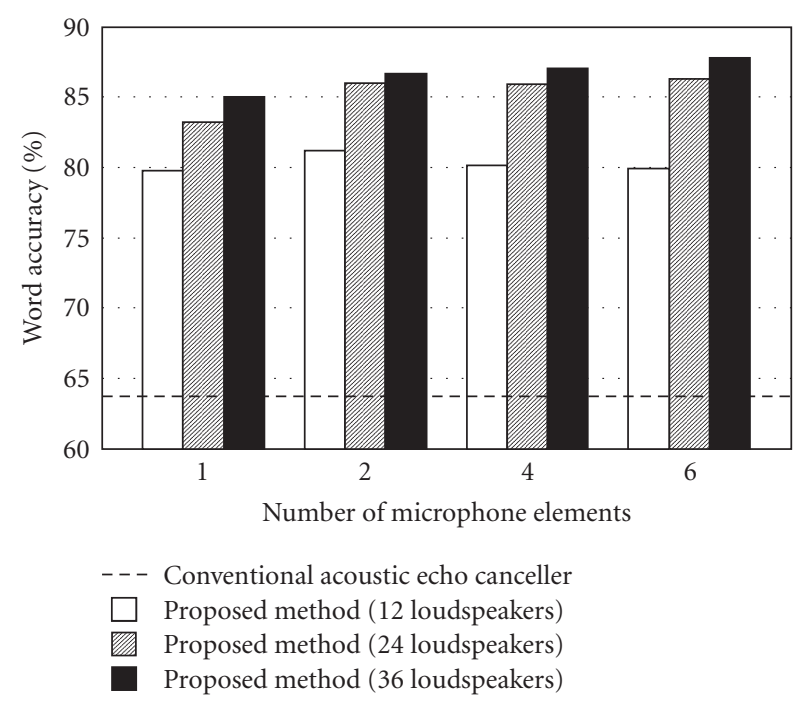

FIgURE 12: Word accuracy when known-noise imposition technique is applied.

$-8.0 \%$ and $-13.2 \%$ without any processing, and $47.1 \%$ and $64.6 \%$ when using the conventional acoustic echo canceller, for the clean model and known-noise imposition, respectively. By masking the redundant component of the response sound, all the results are improved compared with the results using the clean model. All the performances of the proposed method in the figure are superior to those of the conventional acoustic echo canceller. Note that neither system is adapted, that is, optimal weights for system before acoustic change are used. The results show that when the transfer functions are changed, the degradation of speech recognition accuracy can be prevented by increasing the number of transfer channels. From these results, the effectiveness of the proposed response sound elimination technique is ascertained.

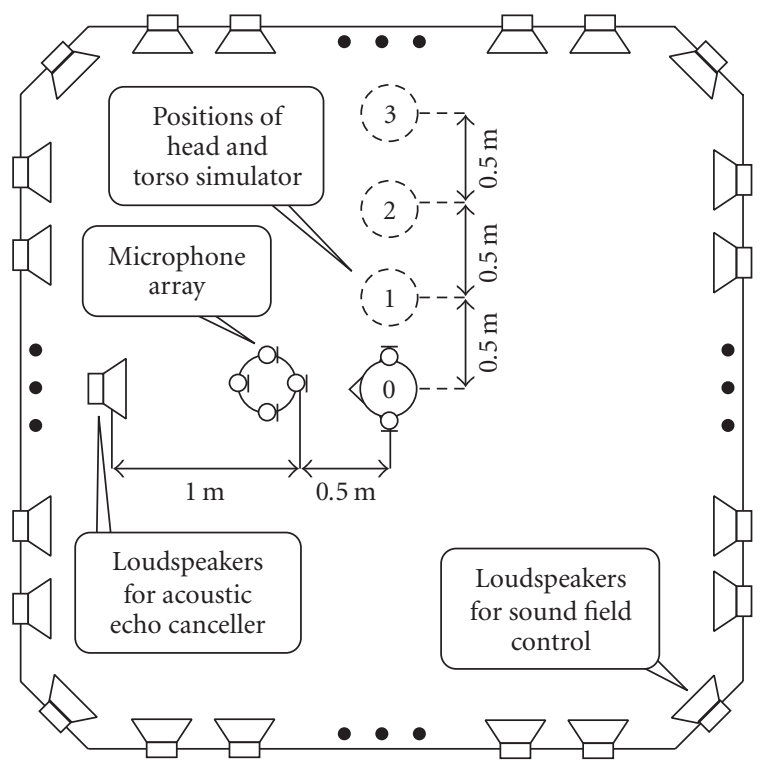

FIGURE 13: Layout of the experimental room in the sound quality assessment.

\section{SOUND QUALITY ASSESSMENT AT VARIOUS USER POSITIONS}

The sound quality of the proposed method is guaranteed and clear sound image is presented only when the user's ears are at the control points where the response sound is reproduced. However, even when the user moves away from the controlled area, the quality of the response sound is sufficient for the spoken dialogue system. To prove this argument, we assess the quality of the response sound which is perceived by the user at various positions. The quality is assessed from two aspects; objective and subjective evaluations.

\subsection{Objective evaluation}

The objective evaluation is carried out via a simulation using impulse responses measured in a real acoustic environment. Figure 13 shows the arrangement of the apparatuses. The room is the same one used in the experiments of Sections 4 and 5. We measured four patterns of impulse responses changing the positions of the HATS from position 0 to position 3. The control points of the MOMNI method are two microphone elements in the microphone array and the ears of the HATS at the position 0 . The primary sound source of the response sound is the loudspeaker of the acoustic echo canceller.

As an evaluation score, we introduce cepstral distance (CD, [23]) which is often used in various speech processings. $\mathrm{CD}$ is given by

$$
\mathrm{CD}(\mathrm{dB})=\frac{1}{F} \sum_{t=1}^{F} \frac{20}{\log 10} \sqrt{\sum_{l=1}^{20} 2\left(C_{\mathrm{obs}}(l, t)-C_{\mathrm{ref}}(l, t)\right)^{2}},
$$




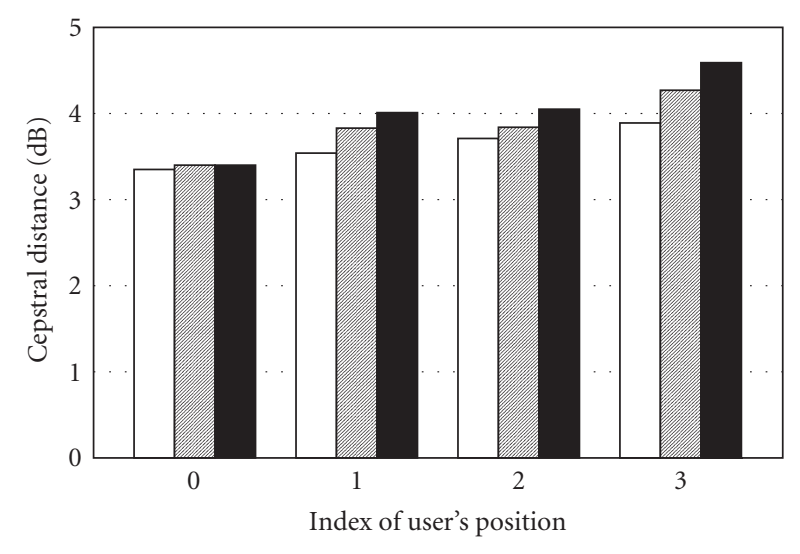

$\square$ Acoustic echo canceller

Proposed method (12 loudspeakers, 1 microphone)

- Proposed method (12 loudspeakers, 2 microphones)

FIgURE 14: Cepstral distance in various positions when 12 loudspeakers are used for the proposed method.

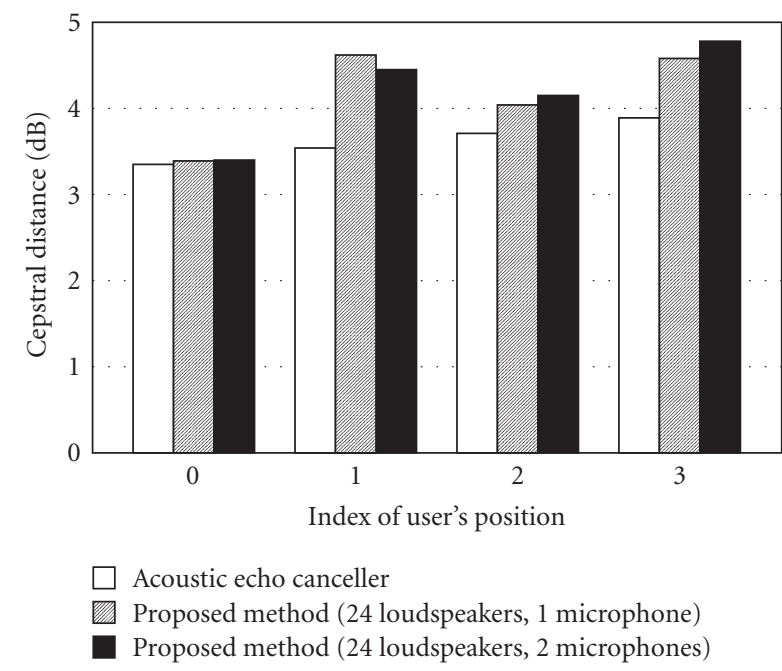

Figure 15: Cepstral distance in various positions when 24 loudspeakers are used for the proposed method.

where $F$ denotes the number of speech frames, $C_{\text {obs }}(l, t)$ is the $l$ th FFT-based cepstrum of the observed signal at the $t$ th frame, and $C_{\text {ref }}(l, t)$ is a reference cepstrum for evaluating the distance. The number of liftering points is 20. A lower $\mathrm{CD}$ value indicates better sound quality. We obtain $C_{\text {ref }}(l, t)$ from the source signal of the response sound. We average the $\mathrm{CDs}$ at both ears. Note that to express $\mathrm{CD}$ in $\mathrm{dB}$, the term 20/log 10 is multiplied to the Eucredian distances between the cepstrum coefficients which are obtained from natural logarithm of the waveforms. In addition, because of symmetry of cepstrum coefficients, we can obtain liftered cepstrum from twice of the cepstrum coefficients from $l=1$ to $l=20$.

Figures 14 and 15 show the CDs of the proposed method compared with those of the acoustic echo canceller. Since

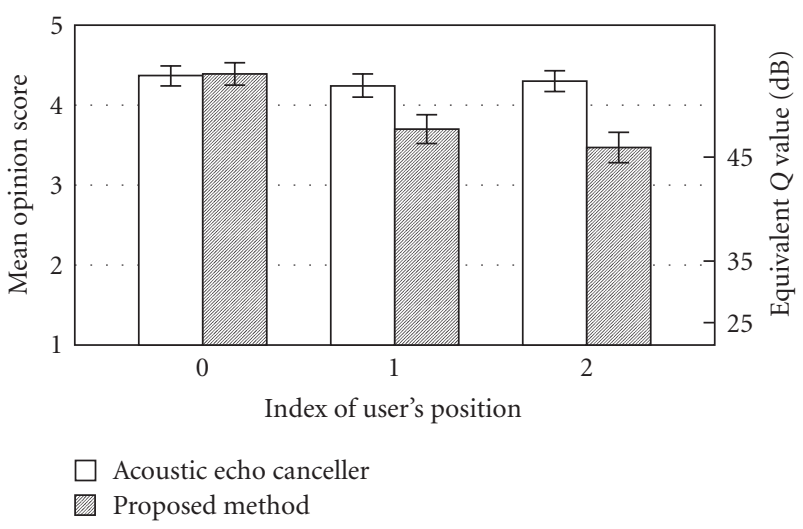

FIGURE 16: Mean opinion score for the positions of the subjects. The blocks show the means and the error bars show the $95 \%$ confidence intervals.

the proposed method reproduces the output sound of the acoustic echo canceller at the position 0 , its CD is similar to that of the acoustic echo canceller. When the HATS is not at the position 0 , the CDs increase. However, its difference is only within $1 \mathrm{~dB}$. Thus, the sound quality degradation of the proposed method is not significant.

\subsection{Subjective evaluation}

To ascertain that the distortion caused by the proposed method is not discomfort, we conduct a subjective evaluation of the sound quality reproduced by the proposed method in a real environment. We changed the positions of the subjects and let them answer mean opinion score (MOS). The opinion score for evaluation was set to a 5-point scale (5: excellent, 4: good, 3: fair, 2: poor, 1: bad).

The room used in this experiment is the same one where the impulse responses are measured in the other experiments. We directed the positions of the subjects by setting chairs at the position 0 , the position 1 , and the position 2 in the Figure 13. The filter of the MOMNI method was designed using measured impulse responses where the HATS is set at the position 0 . The primary sound source of the response sound is the loudspeaker of the acoustic echo canceller. The number of the secondary sound sources is 24 and the microphone elements of the silent reproduction are two.

We compared the MOSs of the proposed method and the acoustic echo canceller. In addition, to give the MOSs objective meaning, we evaluated opinion equivalent $Q$ value [24]. To obtain opinion equivalent $Q$ value, we made three kinds of response sounds imposed white noises whose segmental SNRs are $25 \mathrm{~dB}, 35 \mathrm{~dB}$, and $45 \mathrm{~dB}$. Then these noise-added response sounds are outputted from the acoustic echo canceller. Therefore, the forms of the reproductions are five, that is, the MOMNI method, the acoustic echo canceller, and the three noise-added response sounds. For each of these forms, we prepared 15 sentences of the speech uttered by four males and three females. Then for each of the three positions, we evaluated the MOSs in random orders. 
Figure 16 shows the MOSs for each of the subjects' positions. The scores of the acoustic echo canceller rated at more than four in any of the positions. For the MOMNI method, the score at the position 0 is similar to that of the acoustic echo canceller. Even at the position 0, the binaural response sound is degraded by the difference of the shapes of the head and the sitting heights between the subjects and the HATS. However, we can see that the degradation does not influence the MOSs. Although the MOSs decrease as the subjects move away from the position 0 , the degradation of the score is within one. In addition, even in the worst score at the position 3, the opinion equivalent $Q$ value is over $45 \mathrm{~dB}$. From these findings, it is ascertained that the proposed method can present the response sound with sufficient quality even when the user is out of the prepared position.

\section{CONCLUSION}

We have proposed a barge-in free spoken dialogue interface combining sound field reproduction and a microphone array. It is shown that the response sound elimination performance for the fluctuation of room transfer functions depends on the number of transfer channels. By using an adequate number of loudspeakers and microphone elements, the performance of the proposed method is better than that of the conventional acoustic echo canceller. In the experiment where the proposed method is compared with acoustic echo canceller in the condition that the filter coefficients are fixed, the efficacy of the proposed method is ascertained. Although the proposed method requires multichannel filtering and multiple loudspeakers, the proposed method can maintain the high speech recognition performance in barge-in situation without adaptation.

The remaining problem is that there is still room for improvement in beamforming because the delay-and-sum beamformer is weak against reverberation. We are now addressing this problem via unsupervised adaptive array [25].

\section{APPENDIX}

\section{A. KNOWN-NOISE IMPOSITION}

Even with the use of some effective noise suppression method, it is difficult to eliminate interferencial noises completely. The proposed method is not excepted from this issue and there still exists a residual component of the response sound in the processed signal, because of the fluctuation of the transfer functions. To obtain optimum recognition performance, we generally need to develop matched phoneme models for a speech decoder. However, without a priori information on signal-to-noise ratio, the accurate construction of such matched models is very difficult. To handle many different types of noise, known-noise imposition has been proposed [20]. This technique masks the residual unexpected component with a known noise. To prevent this noise from causing a mismatch in the phoneme feature between the processed signal and the phoneme model, we generate a phoneme model made of the speech imposed with the same

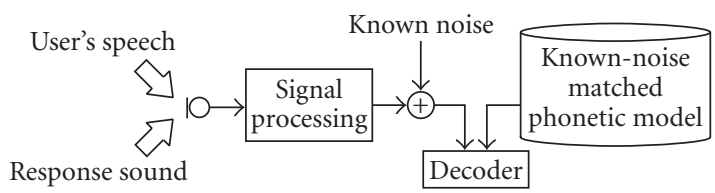

Figure 17: Configuration of known-noise imposition.

noise in advance. We apply this technique in the masking of the residual response sound as follows.

(1) We impose known noise on a speech database and train the corresponding matched model using an EM algorithm in advance.

(2) We impose known noise on the noise-reduced output from the delay-and-sum array in the proposed system.

(3) We perform speech recognition using a known-noise matched model for the system output.

Figure 17 shows a configuration of this process.

\section{ACKNOWLEDGMENTS}

We would like to thank Mr. Koichi Mino of NAIST and Dr. Shoji Makino of NTT CS Laboratories for their valuable discussions. This work was partly supported by CREST Program "Advanced Media Technology for Everyday Living" of JST in Japan.

\section{REFERENCES}

[1] B. H. Juang and F. K. Soong, "Hands-free telecommunications," in Proceedings of International Workshop on Hands-Free Speech Communication, pp. 5-8, Kyoto, Japan, April 2001.

[2] E. Hänsler, "Acoustic echo and noise control: where do we come from-where do we go?" in Proceedings of International Workshop on Acoustic Echo and Noise Control (IWAENC '01), pp. 1-4, Darmstadt, Germany, September 2001.

[3] S. Makino and S. Shimauchi, "Stereophonic acoustic echo cancellation-an overview and recent solutions," in Proceedings of 6th IEEE International Workshop on Acoustic Echo and Noise Control (IWAENC '99), pp. 12-19, Pocono Manor, Pa, USA, September 1999.

[4] Y.-W. Jung, J.-H. Lee, Y.-C. Park, and D.-H. Youn, "A new adaptive algorithm for stereophonic acoustic echo canceller," in Proceedings of IEEE International Conference on Acoustics, Speech and Signal Processing (ICASSP '00), vol. 2, pp. 801-804, Istanbul, Turkey, June 2000.

[5] W. Herbordt and W. Kellermann, "Acoustic echo cancellation embedded into the generalized sidelobe canceller," in Proceedings of European Signal Processing Conference (EUPSICO '00), vol. 3, pp. 1843-1846, Tampere, Finlande, September 2000.

[6] H. Buchner, S. Spors, and W. Kellermann, "Wave-domain adaptive filtering: acoustic echo cancellation for full-duplex systems based on wave-field synthesis," in Proceedings of IEEE International Conference on Acoustics, Speech and Signal Processing (ICASSP '04), vol. 4, pp. 117-120, Montreal, Que, Canada, May 2004.

[7] Y. Tatekura, H. Saruwatari, and K. Shikano, "Sound reproduction system including adaptive compensation of temperature fluctuation effect for broad-band sound control," IEICE Transactions on Fundamentals of Electronics, Communications and Computer Sciences, vol. E85-A, no. 8, pp. 1851-1860, 2002. 
[8] J. Benesty, D. R. Morgan, and J. H. Cho, "A family of doubletalk detectors based on cross-correlation," in Proceedings of 6th IEEE International Workshop on Acoustic Echo and Noise Control (IWAENC '99), pp. 108-111, Pocono Manor, Pa, USA, September 1999.

[9] K. Ochiai, T. Araseki, and T. Ogihara, "Echo canceler with two echo path models," IEEE Transactions on Communications, vol. 25, no. 6, pp. 589-595, 1977.

[10] M. Miyoshi and Y. Kaneda, "Inverse filtering of room acoustics," IEEE Transactions on Acoustics, Speech, and Signal Processing, vol. 36, no. 2, pp. 145-152, 1988.

[11] J. Bauck and D. H. Cooper, "Generalized transaural stereo and applications," Journal of the Audio Engineering Society, vol. 44, no. 9, pp. 683-705, 1996.

[12] Y. Tatekura, H. Saruwatari, and K. Shikano, "An iterative inverse filter design method for the multichannel sound field reproduction system," IEICE Transactions on Fundamentals of Electronics, Communications and Computer Sciences, vol. E84A, no. 4, pp. 991-998, 2001.

[13] S. Haykin, Adaptive Filter Theory, Prentice-Hall, Englewood Cliffs, NJ, USA, 4th edition, 1991.

[14] Y. Suzuki, F. Asano, H.-Y. Kim, and T. Sone, "An optimum computer-generated pulse signal suitable for the measurement of very long impulse responses," Journal of the Acoustical Society of America, vol. 97, no. 2, pp. 1119-1123, 1995.

[15] J. Blauert, Spatial Hearing, MIT Press, Cambridge, Mass, USA, Revised edition, 1997.

[16] J. L. Flanagan, J. D. Johnston, R. Zahn, and G. W. Elko, "Computer-steered microphone arrays for sound transduction in large rooms," Journal of the Acoustical Society of America, vol. 78, no. 5, pp. 1508-1518, 1985.

[17] S. Hayamizu, S. Itahashi, T. Kobayashi, and T. Takezawa, "Design and creation of speech and text corpora of dialogue," IEICE Transactions on Information and Systems, vol. E76-D, no. 1, pp. 17-22, 1993.

[18] A. Lee, T. Kawahara, and K. Shikano, "Julius—an open source real-time large vocabulary recognition engine," in Proceedings of 7th European Conference on Speech Communication and Technology (EUROSPEECH '01), pp. 1691-1694, Aalborg, Denmark, September 2001.

[19] A. Lee, T. Kawahara, K. Takeda, and K. Shikano, "A new phonetic tied-mixture model for efficient decoding," in Proceedings of IEEE International Conference on Acoustics, Speech, and Signal Processing (ICASSP '00), vol. 3, pp. 1269-1272, Istanbul, Turkey, June 2000.

[20] S. Yamade, A. Lee, H. Saruwatari, and K. Shikano, "Unsupervised speaker adaptation based on HMM sufficient statistics in various noisy environments," in Proceedings of 8th European Conference on Speech Communication and Technology (EUROSPEECH '03), vol. 2, pp. 1493-1496, Geneva, Switzerland, September 2003.

[21] K. Itou, M. Yamamoto, K. Takeda, et al., "The design of the newspaper-based Japanese large vocabulary continuous speech recognition corpus," in Proceedings of 5th International Conference on Spoken Language Processing (ICSLP '98), vol. 7, pp. 3261-3264, Sydney, Australia, November-December 1998.

[22] K. Itou, M. Yamamoto, K. Takeda, et al., "JNAS: Japanese speech corpus for large vocabulary continuous speech recognition research," Journal of the Acoustical Society of Japan (E), vol. 20, no. 3, pp. 199-206, 1999.

[23] L. Rabiner and B. H. Juang, Fundamentals of Speech Recognition, Prentice-Hall, Englewood Cliffs, NJ, USA, 1993.
[24] J. R. Deller Jr., J. H. L. Hansen, and J. G. Proakis, Discrete-Time Processing of Speech Signals, Macmillan, New York, NY, USA, 1993.

[25] S. Miyabe, T. Takatani, Y. Mori, H. Saruwatari, K. Shikano, and Y. Tatekura, "Double-talk free spoken dialogue interface combining sound field control with semi-blind source separation," in Proceedings of IEEE International Conference on Acoustics, Speech and Signal Processing (ICASSP '06), vol. 1, pp. 809-812, Toulouse, France, May 2006.

Shigeki Miyabe was born in Nara, Japan, on July 1, 1978. He received the B.E. degree in electrical and electronics engineering from Kobe University in 2003, and received the M.E. degree in information and science from Nara Institute of Science and Technology (NAIST) in 2005. He is now a Ph.D. student at Graduate School of Information Science, NAIST. His research interests include sound field control and array signal processing. He is a Member of the Acoustical Society of Japan (ASJ).

Yoichi Hinamoto received the B.E. degree in electrical and electronic engineering from University of Tokushima in 2001, M.E. degree in information science from NAIST in 2003, and Ph.D. degree in informatics from kyoto University in 2006. He is currently a Research Associate of Takuma National College of Technology. His research interests include digital signal processing and adaptive filter algorithm. He is a Mem-

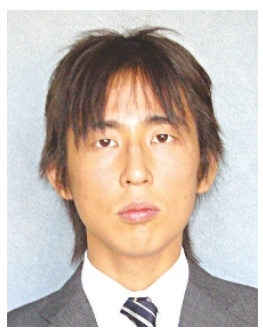
ber of the Institute of Electronics, Information and Communication Engineers of Japan (IEICE) and the Institute of Electrical and Electronics Engineers (IEEE).

Hiroshi Saruwatari was born in Nagoya, Japan, on July 27, 1967. He received the B.E., M.E., and Ph.D. degrees in electrical engineering from Nagoya University, Nagoya, Japan, in 1991, 1993, and 2000, respectively. He joined Intelligent Systems Laboratory, SECOM co., Ltd., Mitaka, Tokyo, Japan, in 1993, where he is engaged in the research and development of the ultrasonic array system for the acoustic imaging. He is cur-

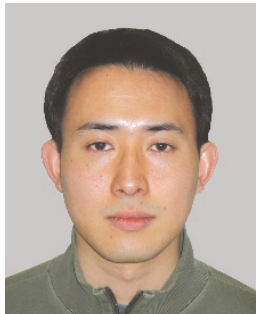
rently an Associate Professor of Graduate School of Information Science, Nara Institute of Science and Technology. His research interests include array signal processing, blind source separation, and sound field reproduction. He received the Paper Awards from IEICE in 2000 and 2006. He is a Member of the IEEE, the VR Society of Japan, the IEICE, and the Acoustical Society of Japan.

Kiyohiro Shikano received the B.S., M.S., and $\mathrm{Ph} . \mathrm{D}$. degrees in electrical engineering from Nagoya University in 1970, 1972, and 1980, respectively. $\mathrm{He}$ is currently a Professor at Nara Institute of Science and Technology (NAIST), where he is directing Speech and Acoustics Laboratory. From 1972 to 1993, he had been working at NTT Laboratories. During 1986-1990,

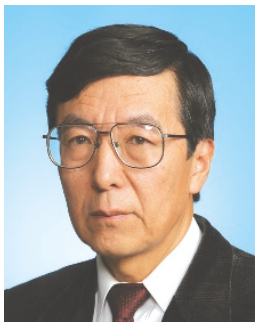


he was the Head of Speech Processing Department at ATR Interpreting Telephony Research Laboratories. During 1984-1986, he was a Visiting Scientist in Carnegie Mellon University. He received the Yonezawa Prize from IEICE in 1975, the Signal Processing Society 1990 Senior Award from IEEE in 1991, the Technical Development Award from ASJ in 1994, IPSJ Yamashita SIG Research Award in 2000, and Paper Award from the Virtual Reality Society of Japan in 2001, IEICE Paper Award in 2005 and 2006, and Inose Award in 2005. He is a Fellow of the Institute of Electronics, Information and Communication Engineers of Japan (IEICE), and Information Processing Society of Japan, and a Member of the Acoustical Society of Japan (ASJ), Japan VR Society, the Institute of Electrical and Electronics Engineers (IEEE), and International Speech Communication Society (ISCA).

Yosuke Tatekura was born in Kyoto, Japan, on May 17, 1975. He received the B.E. degrees in precision engineering from Osaka University in 1998, and received the M.E. and $\mathrm{Ph} . \mathrm{D}$. degrees in information science from Nara Institute of Science and Technology (NAIST) in 2000 and 2002, respectively. He is currently a Research Associate of Shizuoka University. His research interests include sound field control and virtual sound source synthesis.

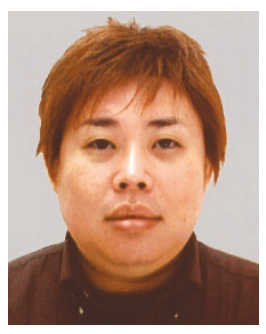

